\title{
Adaptive Parameter Estimation Based Congestion Avoidance Strategy for DTN
}

\author{
Qicai Yang, Futong Qin, Jianquan Bi, Qiubo Xu \\ No. 63880 Troops of PLA \\ Luoyang, China \\ yqc0704@mail.ustc.edu.cn
}

\begin{abstract}
Delay Tolerant Networks are characterized by intermittent connectivity, long delays and often constrained bandwidth. Delay Tolerant Networks often use multi-copy routing schemes for message transmission. Multi-copy routing schemes, such as Epidemic Routing, usually lead to congestion problems. In this paper, we propose an Adaptive Parameter Estimation based congestion Avoidance strategy (APEA) for Delay Tolerant Networks. The APEA strategy includes buffer management policy and packet receiving policy. The buffer management policy is designed for improving the delivery ratio, and the packet receiving policy is designed for reducing the network overhead. The simulation results show that the APEA strategy significantly improves the delivery ratio and reduces the network overhead, compared to the default Drop-FirstReceived strategy.
\end{abstract}

Keywords-DTN; congestion avoidance; buffer management; packet dropping policy

\section{INTRODUCTION}

Delay Tolerant Networks (DTNs) [1] are characterized by intermittent connectivity, long delays and often constrained bandwidth. DTNs represent a broad class of networks such as interplanetary internets, under water sensor networks, and vehicular networks. The characteristics make routing and congestion control become challenges. Then, the DTNRG [2] was created by the IRTF to research DTN, and a new protocol named Bundle Protocol (BP) [3] was introduced to face the challenges. The BP includes a "store and forward" transfer mechanism to meet these challenges. Many new technologies are studied in DTNs, such as new routing protocols, new congestion control strategies. DTNs often use multi-copy routing schemes for message transmission. Multi-copy routing schemes, such as Epidemic Routing [4], usually lead to congestion problems. So congestion control strategies can significantly improve the overall performance of DTNs.

In this paper, we propose the Adaptive Parameter Estimation based congestion Avoidance strategy (APEA) for DTNs. The APEA strategy includes both buffer management policy and packet receiving policy. We implement the APEA strategy in the simulator ONE [5] for evaluating its effects. The simulation uses Random Way Point model (RWP). The APEA strategy is compared with the most used DFR (Drop the First Received packet in the buffer) strategy.

To analyze the general performance of the APEA strategy, we evaluate four performance metrics: delivery ratio, average latency, average hop count and overhead ratio. The simulation results show that the APEA strategy significantly improves the delivery ratio and reduces the network overhead.

The rest part of this paper is organized as follows: section II presents the related works. In section III, we discuss the APEA strategy. Section IV displays simulation results and analyzes the performance of the APEA strategy. Finally, we make a short conclusion in section $\mathrm{V}$.

\section{RELATED WORK}

The DTN characteristics make routing and congestion control become challenges, such as intermittent connectivity, long delays and often constrained bandwidth. DTNs often use multi-copy routing schemes for message transmission. These multi-copy routing schemes often lead to congestion problems, because there are too many copies in the network. Therefore, we need to study congestion control strategies to improve the overall network performance. There are about three categories of congestion control strategies in DTNs.

The first category is congestion avoidance strategies. These strategies generally restraint the message transmission, to decrease the number of message copies in the network. Paper [6] discusses a Token Based Congestion Control mechanism. Network nodes must possess a token in order to inject messages into the network. Tokens are initially uniformly distributed but thereafter move randomly throughout the network. This mechanism results in a reduction in the amount of network transit times. In paper [7], the authors propose a new congestion avoidance mechanism based on path avoidance for DTN. The mechanism optimizes the management of node storage and divides the node state into normal state, congestion adjacent state, congestion state. Based on different node states, separate strategy is employed correspondingly to avoiding congestion.

The second category is flow control strategies. These strategies generally restraint the message flow between neighbors. In paper [8], a hop-by-hop Local Flow Control over InterPlaNetary networks (IPN) is discussed. Its main contribution is the introduction of an asynchronous congestion control mechanism on the network that not presented in the traditional DTN protocol. Through the hopby-hop local flow control, this mechanism tries to resolve buffer overflow, link saturation and useless retransmission problems in order to better utilize the overall system.

The third category is buffer management strategies. When the network becomes congested, different buffer scheduling strategies are deployed for improving the overall performance. In paper [9], authors propose an optimal joint scheduling and drop policy. This policy aims to optimize 
different performance metrics, such as the average delivery rate and the average delivery delay. Paper [10] introduces a novel congestion control strategy, called average forwarding number based on epidemic routing (AFNER). According to the forwarding number queue, the strategy determines the packet forwarding sequence.

In this paper, our APEA strategy includes both buffer management policy and packet receiving policy. The buffer management policy is designed for improving the delivery ratio, and the packet receiving policy for reducing the network overhead.

\section{THE DESIGN OF THE APEA STRATEGY}

In Delay Tolerant Networks, the average latency of network and average hop count are both finite and each is around some value. Thus, when the network becomes congested, we consider dropping packets with more hops and larger latency first. This is the idea of the buffer management policy. But this policy usually cannot observably reduce the network overhead, so we develop the packet receiving policy for reducing most useless transmission in the network. Packets with more hops and larger latency will be received in very low probability. The buffer management policy and the packet receiving policy form our APEA strategy.

\section{A. The Buffer Management Policy}

As mentioned above, when the network becomes congested, we consider dropping packets with more hops and larger latency first. We use the information contained in the node itself to estimate the average latency and the average hop count of network. Then, we use the estimation values to calculate the priority weight of each packet. Packets with more hops and larger latency correspond to smaller priority weights. When the network becomes congested, we drop the packet with smallest priority weight first.

For node $i$ in the network, $m_{i}$ is the number of packets in its buffer, $T_{i j}$ is the living time of packet $j$ in node $i$, and $H_{i j}$ is the hop count of packet $j$ in node $i$. The estimation values and the priority weight are calculated as follows:

1) The average latency estimation

$$
\mathrm{T}_{\mathrm{i}}=\mathrm{C}_{1} \times \frac{1}{\mathrm{~m}_{\mathrm{i}}} \sum_{\mathrm{j}=1}^{\mathrm{m}_{\mathrm{i}}} \mathrm{T}_{\mathrm{ij}}
$$

$C_{1}$ is the latency estimation constant, usually $1 \sim 4$.

2) The average hop count estimation

$$
\mathrm{H}_{\mathrm{i}}=\mathrm{C}_{2} \times \frac{1}{\mathrm{~m}_{\mathrm{i}}} \sum_{\mathrm{j}=1}^{\mathrm{m}_{\mathrm{i}}} \mathrm{H}_{\mathrm{ij}}
$$

$C_{2}$ is the hop count estimation constant, usually $1 \sim 4$.

3) The priority weight function for node $i$

$$
\begin{aligned}
& \mathrm{W}_{\mathrm{i}}=\alpha \times \exp \left(-\mathrm{T}_{p l t} /\left(2 \times \mathrm{T}_{\mathrm{i}}\right)\right) \\
& +(1-\alpha) \exp \left(-\mathrm{H}_{p} \times \mathrm{C}_{\mathrm{ni}} / \mathrm{H}_{\mathrm{i}}\right)
\end{aligned}
$$

$\alpha$ is the latency term coefficient, $\alpha \in(0,1) . \mathrm{T}_{p l t}$ is the current packet living time. $\mathrm{H}_{p}$ is the current packet hop count. $\mathrm{C}_{\mathrm{ni}}$ is the estimation for the average number of neighbor nodes. According to [11], for an Ad Hoc network with $\mathrm{N}$ nodes, the average hop count is usually around $\sqrt{N}$, and the average number of neighbor nodes is around $\ln (N)$. Thus, we get the estimation for $\mathrm{C}_{\mathrm{ni}}$ :

$$
\mathrm{C}_{\mathrm{ni}}=2 \ln \left(\mathrm{H}_{\mathrm{i}}\right)
$$

The coefficients $C_{1}, C_{2}$ and $\alpha$ do affect the performance of APEA. These coefficients can be set to different values for different scenarios. Usually, we set $C_{1}=2.5, C_{2}=2.5$ and $\alpha=0.5$, if there is no special request.

Though the formulas, we can find that the calculating process just use the information contained in the node itself, so this policy can be deployed in various scenarios.

\section{B. The Packet Receiving Policy}

The buffer management policy usually cannot observably reduce the network overhead, so we develop the packet receiving policy for reducing most useless transmission in the network. Packets with more hops and larger latency, which correspond to smaller priority weights, will be received in lower probability. Thus, the packet receiving policy directly reduces the network traffic. The designed packet receiving policy is described as follows:

1) If the buffer using rate is lower than $r_{t}\left(0<r_{t}<1\right)$, we consider that the network is not in the congestion state, and there is nothing more to do.

2) If the buffer using rate equals to $r_{t}$ or is larger than $r_{t}$, we consider that the network is in the congestion state. If the destination of the coming packet is the current node, this packet must be received.

3) If the buffer using rate equals to $r_{t}$ or is larger than $r_{t}$, we consider that the network is in the congestion state. If the destination of the coming packet is not the current node, The following mechanism will work:

We calculate the average priority weight $W_{A V G}$ and the maximal priority weight $W_{\text {MAX }}$ in the current node. Then we calculate the priority weight $W_{T B C}$ of the coming packet (in the $W_{T B C}$ calculation, the hop count $H_{p}$ plus 1$)$. We compare $W_{T B C}$ with $W_{A V G}$ and $W_{M A X}$ :

a) if $W_{T B C}<W_{A V G}$, the current node will refuse to receive the coming packet;

b) if $W_{T B C}>W_{M A X}$, the current node must receive the coming packet;

c) if $W_{A V G} \leqslant W_{T B C} \leqslant W_{M A X}$, the current node will receive the coming packet in probability $p$ : 


$$
p=\frac{W_{T B C}-W_{A V G}}{W_{M A X}-W_{A V G}}
$$

When receiving the new packet, if there is not enough free space for the new packet, the current node will free enough space according to the buffer management policy. Usually, $r_{t}$ has little effect on the network performance, and it is suitable to set $r_{t}=0.8$.

Notice that, in step 3), when $W_{T B C}<W_{A V G}$, the current node will refuse to receive the coming packet. This means, when the network is in the congestion state, this policy will at least reduce half of the network traffic. This is designed for multi-copy routing schemes. Because there are multiple copies of packets in the network, reducing half of the network traffic will not reduce the delivery ratio. Contrarily, it will help improving the delivery ratio when in congestion state.

\section{Simulation Results AND ANALysis}

The ONE simulator is used for our simulation. We employ Epidemic Routing protocol to do the simulation. In the results of the simulation, we focus on delivery ratio, average latency, average hop count and overhead ratio. The delivery ratio and overhead ratio are defined as follows: delivery_ratio $=$ delivered $/$ created

overhead_ratio $=($ relayed-delivered $) /$ delivered

The "delivered" is the number of packets received by destination nodes. The "created" is the number of packets created by source nodes. The "relayed" is the total number of packets relayed in the network. The average latency is average End-to-End latency of "delivered" packets, and the average hop count is the average hop count of "delivered" packets too.

There are two scenarios in our simulation. The first scenario is the Ad Hoc scenario. There is only one group of nodes in the Ad Hoc scenario. The settings of every node are the same. The second scenario is the city scenario. This scenario simulates the Helsinki city. There are six groups of nodes in the scenario. Different settings are deployed for different groups. In the following experiments, we set $C_{1}=2.5, C_{2}=2.5, \alpha=0.5$, and $r_{t}=0.8$.

\section{A. The Ad Hoc Scenario}

In this scenario, we assume that all the nodes are with the same communication ranges, and when every two nodes are in communication range, they can setup the connection, and stop transferring when they are out of communication range. There are ten rounds in the simulation. The node buffer size is $1 \mathrm{MB}$ for the first round and $2 \mathrm{MB}$ for the second round, and so on. The concrete parameter settings are list in table I.

\begin{tabular}{|l|l|}
\hline \multicolumn{1}{|c|}{ Settings } & \multicolumn{1}{c|}{ Values } \\
\hline Transmission range & $50 \mathrm{~m}$ \\
\hline Message size & $100 \sim 250 \mathrm{~KB}$ \\
\hline Message TTL & $60 \mathrm{~min}$ \\
\hline Routing protocol & Epidemic Routing \\
\hline Scenario size & $1000 \mathrm{~m} \times 1000 \mathrm{~m}$ \\
\hline Number of nodes & 50 \\
\hline Simulation time & $20 \mathrm{~h}$ \\
\hline Message creation interval & One new message every 8 to $12 \mathrm{~s}$ \\
\hline
\end{tabular}

We compare the APEA strategy with the most used DFR strategy. The experiment results in the Ad Hoc scenario are shown as follows:

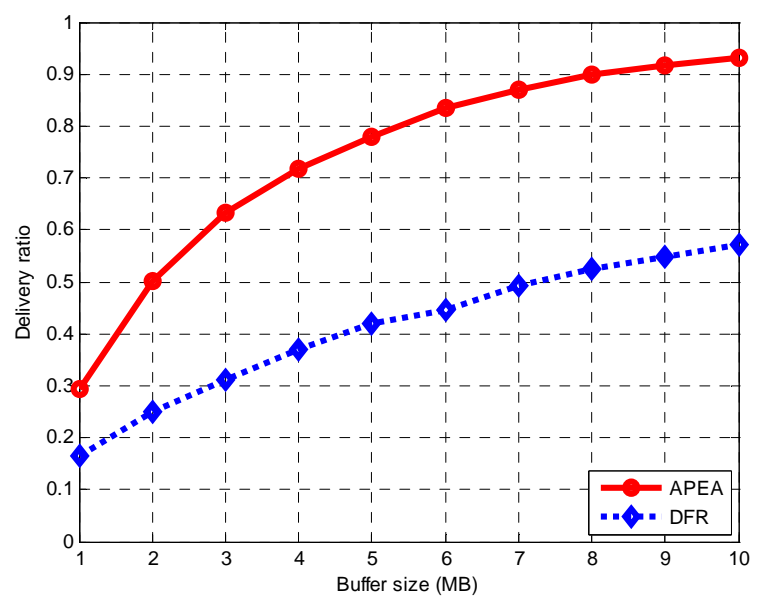

Figure 1. Delivery ratio performance in the Ad Hoc scenario

From Figure 1, we can find that our APEA strategy significantly improves the delivery ratio. Compared to the DFR strategy, the average improvement of the delivery ratio of the APEA strategy is around $77 \%$. When the buffer size is $4 \mathrm{MB}$, compared to the DFR strategy, the best improvement of the delivery ratio of the APEA strategy is about $94 \%$. These results are mainly because APEA is based on better buffer management policy and better packet receiving policy.

TABLE I. SETtings OF THE AD Hoc ScEnARIO

\begin{tabular}{|l|l|}
\hline \multicolumn{1}{|c|}{ Settings } & \multicolumn{1}{c|}{ Values } \\
\hline Node speed & $0.5 \sim 1.5 \mathrm{~m} / \mathrm{s}$ \\
\hline Transmission speed & $250 \mathrm{~KB} / \mathrm{s}$ \\
\hline
\end{tabular}




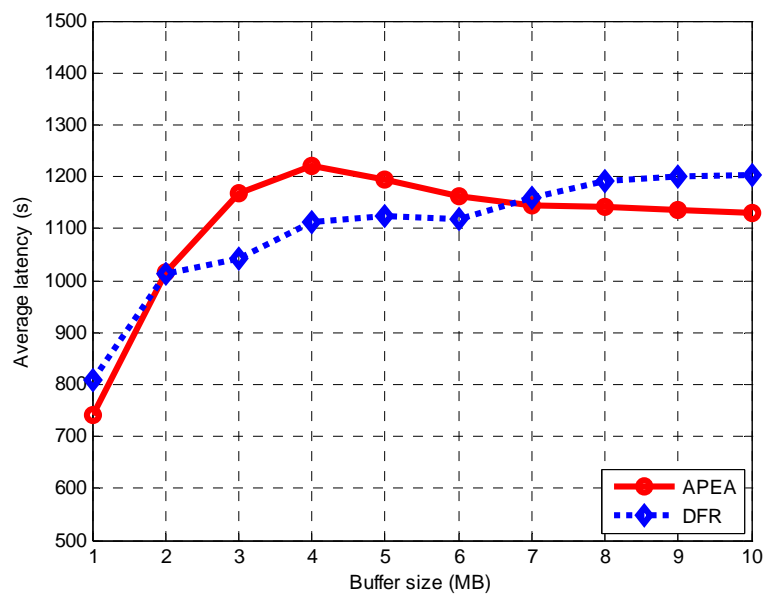

Figure 2. Average latency performance in the Ad Hoc scenario

From Figure 2, we can find that the average latency performance of the APEA strategy is similar to the DFR strategy. The average latency performance of the APEA strategy is not good enough, because the latency estimation value in formula (1) is not stable. When the simulation time increases, even if there is no transmission in the network for a while, the latency estimation value in formula (1) increases too, so the average latency performance of the APEA strategy is not very well.

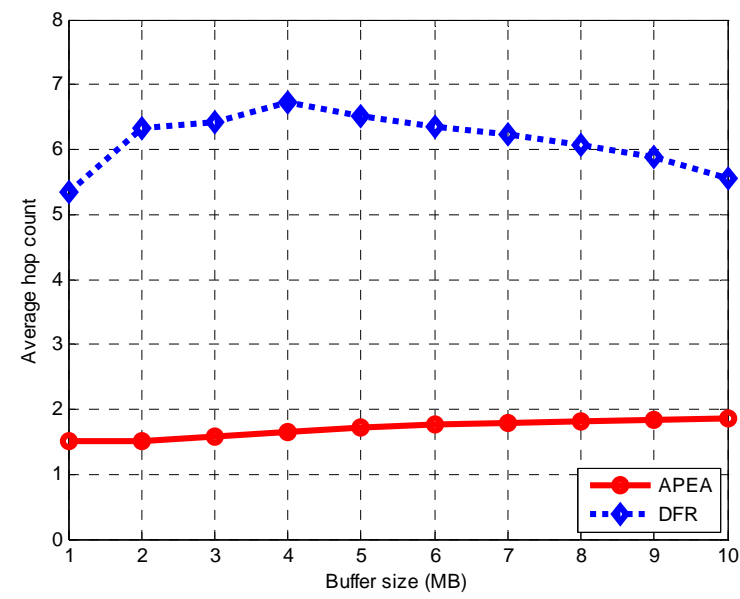

Figure 3. Average hop count performance in the Ad Hoc scenario

From Figure 3, we can find that the average hop count performance of the APEA strategy is better than the DFR strategy. This is because the APEA strategy drops packets with more hops and larger latency first. The hop count estimation value in formula (2) is unlike the latency estimation value. If there is no transmission in the network for a while, the hop count estimation value will not change.

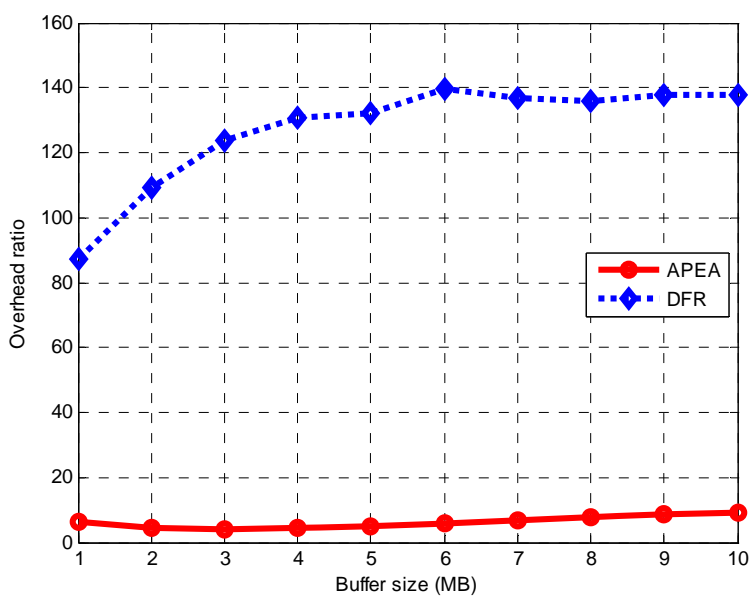

Figure 4. Overhead ratio performance in the Ad Hoc scenario

From Figure 4, we can find that the APEA strategy gains lesser overhead ratio, compared to the DFR strategy. The overhead ratio of the APEA strategy is around 10, and the overhead ratio of the DFR strategy is around 120 . The overhead ratio performance of the APEA strategy is very good, because the packet receiving policy of the APEA strategy reduces most useless transmission in the network. The APEA strategy makes nodes just receive "meaningful" packets, which are with less hops and lower latency.

These experiment results in the Ad Hoc scenario show that: the APEA strategy significantly improves the delivery ratio and reduces the overhead ratio, compared to the DFR strategy. The average hop count performance of APEA is good, but the average latency performance of APEA is not very well.

\section{B. The City Scenario}

The city scenario is totally the default scenario in the ONE simulator. The city scenario is more complex than the Ad Hoc scenario. The city scenario simulates the Helsinki city. There are six groups of nodes in the network. Pedestrians (group 1 and group 3) can move anywhere in the map, but cars (group 2) can only move on roads. There are three groups of trams in the city scenario, group 4 is with broadcast interfaces, and group 5 and group 6 are normal. Most of the nodes in the network are pedestrians and cars. There are ten rounds in the simulation. For group 1, group 2 and group 3 , the node buffer size is $1 \mathrm{MB}$ for the first round and $2 \mathrm{MB}$ for the second round, and so on. For the groups of trams, the node buffer size is always $50 \mathrm{MB}$. The concrete parameter settings are list in table II.

TABLE II. SETTINGS OF THE CITY SCENARIO

\begin{tabular}{|l|l|}
\hline \multicolumn{1}{|c|}{ Settings } & \multicolumn{1}{c|}{ Values } \\
\hline & Group 1: $0.5 \sim 1.5 \mathrm{~m} / \mathrm{s}, 40$ pedestrians \\
& Group 2: $2.7 \mathrm{~m} / \mathrm{s} \sim 13.9 \mathrm{~m} / \mathrm{s}, 40$ cars only \\
on roads & Group 3: $0.5 \mathrm{~m} / \mathrm{s} \sim 1.5 \mathrm{~m} / \mathrm{s}, 40$ pedestrians \\
& Group 4: $7 \mathrm{~m} / \mathrm{s} \sim 10 \mathrm{~m} / \mathrm{s}, 2$ trams with \\
& broadcast interfaces \\
\hline
\end{tabular}




\begin{tabular}{|l|l|}
\hline \multicolumn{1}{|c|}{ Settings } & \multicolumn{1}{c|}{ Values } \\
\hline & $\begin{array}{l}\text { Group } 5: 7 \mathrm{~m} / \mathrm{s} \sim 10 \mathrm{~m} / \mathrm{s}, 2 \text { trams } \\
\text { Group } 6: 7 \mathrm{~m} / \mathrm{s} \sim 10 \mathrm{~m} / \mathrm{s}, 2 \text { trams }\end{array}$ \\
\hline Transmission speed & $250 \mathrm{~KB} / \mathrm{s}(10 \mathrm{MB} / \mathrm{s}$ for group 4$)$ \\
\hline Transmission range & $10 \mathrm{~m}(1000 \mathrm{~m}$ for group 4$)$ \\
\hline Message size & $500 \mathrm{~KB} \sim 1 \mathrm{MB}$ \\
\hline Message TTL & $300 \mathrm{~min}$ \\
\hline Routing protocol & Epidemic Routing \\
\hline Scenario size & $4500 \mathrm{~m} \times 3400 \mathrm{~m}$ \\
\hline Number of nodes & 126 \\
\hline Simulation time & $12 \mathrm{~h}$ \\
\hline Message creation interval & One new message every 25 to $35 \mathrm{~s}$ \\
\hline
\end{tabular}

We compare the APEA strategy with the most used DFR strategy. The experiment results in the city scenario are shown as follows:

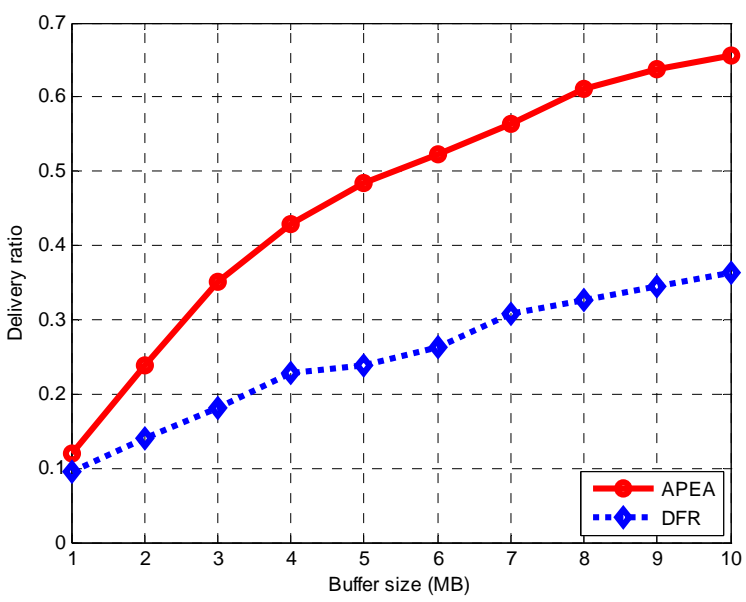

Figure 5. Delivery ratio performance in the city scenario

From Figure 5, we can find that, when the buffer size becomes larger, our APEA strategy significantly improves the delivery ratio. Compared to the DFR strategy, the average improvement of the delivery ratio of the APEA strategy is around $86 \%$. When the buffer size is $5 \mathrm{MB}$, compared to the DFR strategy, the best improvement of the delivery ratio of the APEA strategy is about $103 \%$. These results are mainly because APEA is based on better buffer management policy and better packet receiving policy. When the buffer size is $1 \mathrm{MB}$, the improvement is not obvious, because there are so few packets (usually one or two) in the buffer to use for the latency estimation and the hop count estimation. When packets in the buffer are too few, the APEA strategy will not work well.

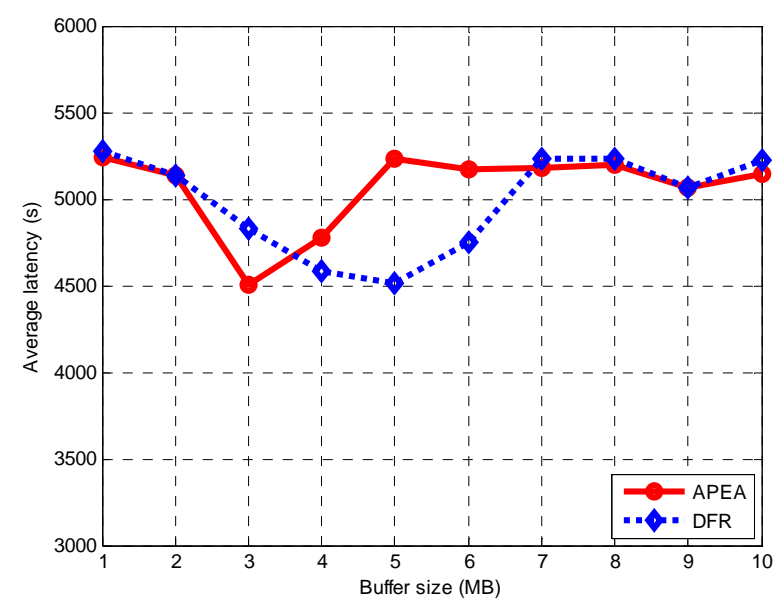

Figure 6. Average latency performance in the city scenario

From Figure 6, we can find that the average latency performance of the APEA strategy is similar to the DFR strategy. The average latency performance of the APEA strategy is not good enough, because the latency estimation value in formula (1) is not stable. When the simulation time increases, even if there is no transmission in the network for a while, the latency estimation value in formula (1) increases too, so the average latency performance of the APEA strategy is not very well.

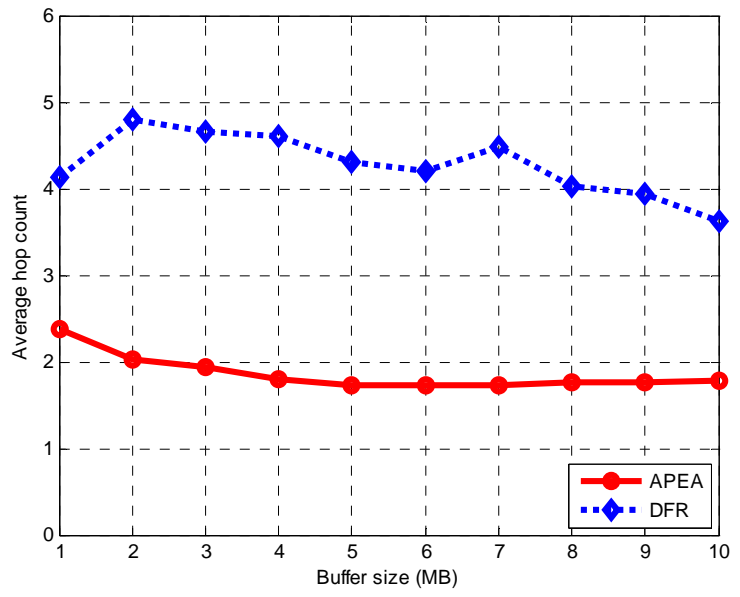

Figure 7. Average hop count performance in the city scenario

From Figure 7, we can find that the average hop count performance of the APEA strategy is better than the DFR strategy. This is because the APEA strategy drops packets with more hops and larger latency first. The hop count estimation value in formula (2) is unlike the latency estimation value. If there is no transmission in the network for a while, the hop count estimation value will not change. 


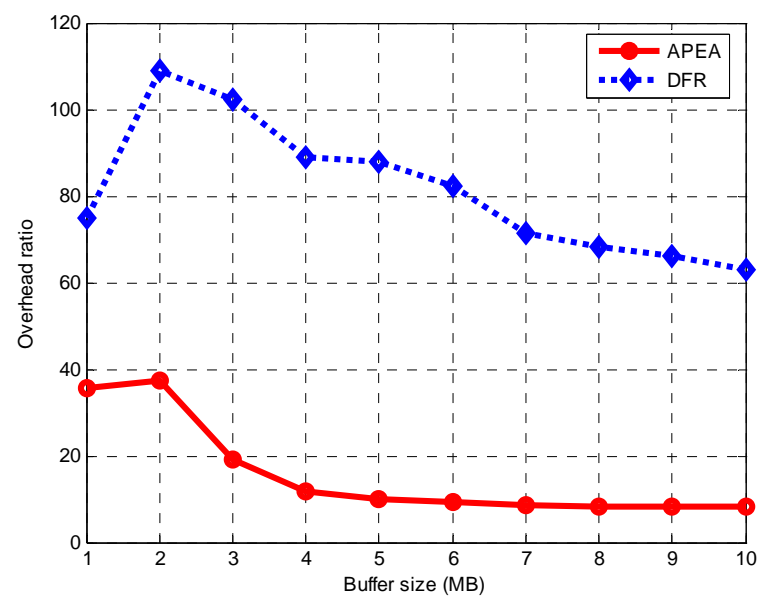

Figure 8. Overhead ratio performance in the city scenario

From Figure 8, we can find that the APEA strategy gains lesser overhead ratio, compared to the DFR strategy. When the buffer size is $1 \mathrm{MB}$, the APEA strategy reduces about half of the overhead ratio. Because the packet receiving policy at least reduces half of the network traffic, even if the improvement of delivery ratio is not obvious, APEA still significantly reduces the overhead ratio. The overhead ratio performance of the APEA strategy is very good, because the packet receiving policy of the APEA strategy reduces most useless transmission in the network. The APEA strategy makes nodes just receive "meaningful" packets, which are with less hops and lower latency.

These experiment results in the city scenario show that: the APEA strategy significantly improves the delivery ratio and reduces the overhead ratio, compared to the DFR strategy. But when there are too few packets in the buffer, the improvement of delivery ratio of APEA is not obvious. The average hop count performance of APEA is good, but the average latency performance of APEA is not very well.

\section{CONCLUSION}

Delay Tolerant Networks often use multi-copy routing schemes for message transmission. Multi-copy routing schemes, such as Epidemic Routing, usually lead to congestion problems. In this paper, we present a new congestion avoidance strategy APEA to solve congestion problems. The simulation results show that the APEA strategy significantly improves the delivery ratio and reduces the network overhead. The average hop count performance of APEA is good too. It is our future work to improve the APEA strategy for reducing the network latency.

\section{REFERENCES}

[1] K. Fall, "A delay tolerant network architecture for challenged internets”, in Proc. ACM SIGCOMM, pp.27-24, 2003.

[2] DTN research group homepage[2012.6/2010.6], www.DTNrgp.org.

[3] K. Scott, S. Burleigh, RFC5050: Bundle Protocol Specification, November 2007
[4] A. Vahdat and D. Becker, "Epidemic routing for partially connected ad hoc networks,” Technical Repor t CS-200006, Tech. Rep., 2000.

[5] The Opportunistic Network Environment simulator homepage [2012.6/2010.6], www.netlab.tkk.fi/tutkimus/dtn/theone/

[6] E. Coe, C. Raghavendra, "Token Based Congestion Control for DTNs", IEEE Aerospace Conference, pp. 1-7, 2010

[7] Daowen Hua , Xuehui Du , Lifeng Cao , Guoyu Xu , Yanbin Qian , "A DTN congestion avoidance strategy based on path avoidance", 2nd International Conference on Future Computer and Communication (ICFCC), vol. 1, pp. 855-860, 2010

[8] F. De Rango, M. Tropea, G.B. Laratta, S. Marano, "Hop-by-Hop Local Flow Control over InterPlaNetary Networks based on DTN Architecture” , IEEE International Conference on Communications, ICC '08, pp. 1920 - 1924, 2008.

[9] A. Krifa, C. Barakat, T. Spyropoulos, "An optimal joint scheduling and drop policy for Delay Tolerant Networks", World of Wireless, Mobile and Multimedia Networks, pp: 1-6, 2008.

[10] L. Yun, C. Xinjian, L. Qilie, Y. Xiaohu, "A Novel Congestion Control Strategy in Delay Tolerant Networks”, Second International Conference on Future Networks, ICFN '10, pp. 233 - 237, 2010.

[11] P. Gupta, P.R. Kumar, “The capacity of wireless networks”, IEEE Transactions on Information Theory, pp.388 - 404, 2000 